\title{
The biological roots of music and dance: Extending the credible signaling hypothesis to predator deterrence
}

\author{
Edward H. Hagen \\ Department of Anthropology \\ Washington State University \\ edhagen@wsu.edu
}

Published in Human Nature: https://doi.org/10.1007/s12110-022-09429-9

\begin{abstract}
After they diverged from panins, hominins evolved an increasingly committed terrestrial lifestyle in open habitats that exposed them to increased predation pressure from Africa's formidable predator guild. In the Pleistocene, Homo transitioned to a more carnivorous lifestyle that would have further increased predation pressure. An effective defense against predators would have required a high degree of cooperation by the smaller and slower hominins. It is in the interest of predator and potential prey to avoid encounters that will be costly for both. A wide variety of species, including carnivores and apes and other primates, have therefore evolved visual and auditory signals that deter predators by credibly signaling detection and/or the ability to effectively defend themselves. In some cooperative species, these predator deterrent signals involve highly synchronized visual and auditory displays among group members. Hagen \& Bryant (2003) proposed that synchronized visual and auditory displays credibly signal coalition quality. Here, this hypothesis is extended to include credible signals to predators that they have been detected and would be met with a highly coordinated defensive response, thereby deterring an attack. Within-group signaling functions are also proposed. The evolved cognitive abilities underlying these behaviors were foundations for the evolution of fully human music and dance.
\end{abstract}




\section{Introduction}

Music and dance are vocal and motor displays that, compared to most such displays in nonhuman animals, have an unusual feature: they often involve a high degree of temporal synchrony among multiple performers. Synchronization is achieved by entrainment to a beat, i.e., rhythm. According to one scheme, this entails motor periodicity (quasi-periodic repetitive actions), beat extraction (inference of an isochronic pulse from repetitive stimuli), audiomotor entrainment (synchronizing motor actions to an inferred beat), and meter (hierarchical structuring of beats) (Kotz, Ravignani, \& Fitch, 2018). These specialized abilities indicate adaptation. Although synchronized vocal, visual, and/or motor displays by more than two individuals are rare in non-human animals, they are not unknown. ${ }^{1}$ Such displays, termed synchronous chorusing (which we will restrict to displays by three or more individuals) are exhibited by fireflies, crickets, frogs, and birds (Ravignani, Bowling, \& Fitch, 2014).

Comparative analysis of synchronous chorusing in other species could elucidate the evolutionary explanation for human music and dance. The evolutionary explanation for synchronous chorusing in non-human animals is still under debate, however, and might vary across species. Synchronous chorusing might improve mate attraction, predator confusion, or self-defense (the selfish herd), or it could be an epiphenomenon of competitive signaling (Merker, Madison, \& Eckerdal, 2009; Ravignani et al., 2014). If synchronous chorusing is a human adaptation, it must have evolved in response to an important selection pressure in the human lineage. Merker (1999), for example, proposed that because early hominins were probably male philopatric with female dispersal, males evolved to cooperatively produce loud auditory calls to attract dispersing females (see also Merker et al., 2009). Although this is an intriguing idea, the chimpanzee and bonobo lineages, which have the same dispersal patterns, would also have experienced this selection pressure yet did not evolve this strategy. It is therefore worth considering alternative explanations. In particular, a few spectacular examples of synchronized motor displays in bees, fish, and birds appear to have evolved to deter predators, raising the possibility that increased predation pressure in the human lineage helped select for synchronous chorusing.

This paper is organized as follows. First, as argued by many authors, the case will be made that the human lineage experienced an increase in predation pressure, especially when human ancestors adopted a more carnivorous lifestyle. Second, theory and evidence will be presented that, to deter attacks, prey often evolve to signal predators. Third, non-human examples of synchronized signaling by prey to predators will be described. Finally, the coalition quality hypothesis for the evolution of music and dance (Hagen \& Bryant, 2003; Hagen \& Hammerstein, 2009; Mehr, Krasnow, Bryant, \& Hagen, 2021) will be extended to synchronized signaling by hominins to predators, as suggested by Jordania (2008), and additional credible signaling functions will be introduced. Alternative evolutionary

${ }^{1}$ Synchronized displays by two individuals, termed duets, are more common. For a review of duetting in birds, see Hall (2009). 
accounts of human music and dance, such as the auditory cheesecake, social bonding, and sexual selection hypotheses, have been extensively reviewed and critiqued elsewhere (Mehr et al., 2021; Savage et al., 2021) and will not be discussed here.

\section{Primate terrestriality and increased predation pressure}

Predator-prey co-evolution was a key driver of the evolution of the nervous system and the diversification of animals over half a billion year ago (Kristan, 2016; Monk \& Paulin, 2014; Paulin \& Cahill-Lane, 2019). Predators impact prey via direct predation and through the fitness costs of morphological and behavioral antipredator responses. Behavioral responses include changes in habitat use, vigilance, foraging, aggregation, movement patterns, and sensitivity to environmental conditions (Creel \& Christianson, 2008).

Primates have long been preyed upon (Hart \& Sussman, 2008). The evolution of primate sociality c. 52 million years ago (Mya) is believed to have mitigated the increased risk of predation that occurred with the transition to diurnality around the same time (Shultz, Opie, \& Atkinson, 2011). Arboreality is primitive in primates, and many defining primate traits, such as grasping extremities, are interpreted as adaptations to an arboreal way of life, perhaps linked to the diversification of the angiosperms (Eriksson, 2016; Sussman, Tab Rasmussen, \& Raven, 2013). The many primate species that subsequently evolved to travel, feed, or dwell terrestrially, including our hominin ancestors, are thought to have been under higher predation pressure. Indirect evidence for increased predation pressure on terrestrial primates includes that they generally have larger body sizes and live in larger groups than arboreal species, traits that are interpreted as predator defenses (e.g., Willems \& van Schaik, 2017). Direct evidence for the hypothesis is mixed, however. In one study, primate predation rates were not associated with group sizes or body sizes; instead, they fluctuated with the diel cycle, with smaller vervets more vulnerable to leopard attacks during the day and larger baboons more vulnerable at night (Isbell, Bidner, Van Cleave, Matsumoto-Oda, \& Crofoot, 2018).

Predation rates, however, reflect both predation pressure and coevolved antipredator strategies. Many studies have therefore investigated primate perceptions of varying predation risk across their home range and their corresponding responses, using a concept known as the landscape of fear (Gaynor, Brown, Middleton, Power, \& Brashares, 2019). In two related studies of sympatric vervet and samango monkeys preyed on by leopards, baboons, and eagles, for instance, intensity of monkey range use was more influenced by their perceived predation risk, indexed by alarm calls, than it was by resource distribution (Coleman \& Hill, 2014; Willems \& Hill, 2009). The most convincing studies of the relationship between terrestriality and predation risk have utilized natural experiments involving different populations of the same primate species that have greater or lesser exposure to terrestrial predators. Capuchins living on islands devoid of predators exploited terrestrial niches as much as 200 times more frequently than mainland capuchins who were exposed to terrestrial predators, for example, a pattern that suggests predation risk strongly deters terrestriality (Monteza-Moreno, Crofoot, Grote, \& Jansen, 2020). 


\section{Hominin terrestriality and increased predation pressure}

Humans are apes. Extant non-human apes are large-bodied primates inhabiting African and southeast Asian tropical forests that are characterized by their lack of an external tail, high joint mobility, an orthograde (upright) body plan, and, in great apes, larger brains and slower life history profiles than other non-human primates (Almécija et al., 2021). Apes diverged from old world monkeys in the Oligocene (34-23 mya) and then diversified into more than 50 genera spread across much of Africa and Eurasia in the Miocene (23-5.3 mya). In one account, apes evolved in intense competition with monkeys over food, with monkeys specializing to acquire less ripe fruit near the tree core and apes evolving suspensory locomotion to acquire the riper remaining fruit near the canopy periphery (Hunt, 2016).

Through the first half of the Miocene, much of the planet was heavily forested, providing the niches that the poorly understood Miocene apes presumably occupied. From the middle to the late Miocene, however, perhaps in response to a cooling and aridifying climate, forests diminished and grasslands expanded (Couvreur et al., 2021; Steinthorsdottir et al., 2021), so much so that by the late Miocene there might have been an immense connected savanna biome across much of Africa and Eurasia (Kaya et al., 2018). In the late Miocene, 69 mya, the human and chimpanzee lineages diverged from a common (presumably African) ape ancestor (Püschel, Bertrand, O’Reilly, Bobe, \& Püschel, 2021; Reis et al., 2018). As the climate continued to cool, grasslands continued to expand through the Pliocene (5.3-2.6 mya) in Africa (but disintegrate in Eurasia, Kaya et al., 2018).

There are multiple models of the last common ancestor (LCA) of panins (chimpanzees and bonobos) and hominins. The most influential model is based on extant African great apes, especially panins, which locomote by knuckle walking, a compromise that enables terrestrial travel while retaining climbing adaptations. On this view, hominins originated from an ancestor that was already terrestrial while traveling (Almécija et al., 2021) and therefore subject to greater predation, possibly selecting for the larger body size seen in extant Africa great apes. A second group of models proposes that the LCA was a late Miocene ape whose locomotion involved various arboreal and terrestrial behaviors such as palmigrade quadrupedalism, climbing and suspension, vertical climbing, or arboreal bipedalism and suspension. On this view, The LCA was smaller and more arboreal, and greater terrestriality and body size evolved independently in hominins and African great apes (Almécija et al., 2021).

Either way, human ancestors increasingly inhabited open African grasslands, evolving larger post-canine dentition and reduced canines, likely to better exploit grassland plant resources, but retaining ape-sized brains. Importantly, there was a profound change in hominin locomotion, i.e., bipedalism, that indicates a transition to a more committed terrestrial lifestyle (Foley, 2016), arguably intensifying predation pressure.

To estimate changes in primate antipredator responses associated with the transition from a more arboreal lifestyle in wooded habitats to a more terrestrial lifestyle in open habitats, Willems \& van Schaik (2017) assembled data on the modal group size, group composition, and degree of sexual dimorphism for a large number of social primate species that were 
classified as [semi-] terrestrial or arboreal, and as inhabiting wooded or more open vegetation. This resulted in three broad lifestyle categories: terrestrial species that live in relatively open habitats, terrestrial species that live in wooded habitats, and arboreal species that live in wooded habitats. They then surveyed the literature for reports on counter-attacks by primate prey against predator species, recording the species of primate and predator, the age-sex class and number of individuals involved, and whether or not the predator was killed or died as a consequence of the counter-attack. They found more counter-attacks than expected by primate species living in open terrestrial habitats, mostly by males, and fewer than expected in species living in wooded arboreal habitats. They also found that, compared to species in wooded arboreal habitats, species in terrestrial open habitats had larger group sizes, more males in the group, and greater sexual dimorphism. Critical for the hypothesis that will be advanced here, male primates often cooperated in counter-attacks against terrestrial carnivores. From these results, Willems \& van Schaik (2017) infer that in response to increased predation risk from the transition to a more open terrestrial habitat, hominins lived in larger groups with many larger males who cooperated in predator defense.

\section{Carnivory in Homo and increased predation pressure}

The first members of our genus, Homo, appeared in Africa around the end of the Pliocene and beginning of the Pleistocene, c. 2.6 mya (Antón, Potts, \& Aiello, 2014). A range of evidence, including stone tool cut marks on bones, life history changes, and divergent modern hunter-gatherer vs. chimpanzee diets, leave little doubt that from the early through the mid Pleistocene, vertebrate meat was an increasingly important component of Homo diets (Antón et al., 2014; Ben-Dor, Sirtoli, \& Barkai, 2021; Finch \& Stanford, 2004; Pontzer \& Wood, 2021). Considerably more contentious is the timing, extent, and mode of meat acquisition by early Homo, such as the importance of scavenging (Pobiner, 2020) vs. hunting (Domínguez-Rodrigo et al., 2021).

Regardless of the mode of meat acquisition, Homo would not only have been potential prey itself, it would also have contended with competition for carcasses from members of Africa's formidable predator guild. There is intense intraguild competition among carnivores. In Africa, lions and spotted hyenas, the two largest carnivores, scavenge kills from each other and from smaller carnivores like leopards, cheetahs, and wild dogs. Male lions are responsible for $20-50 \%$ of spotted hyena deaths, and interestingly, do not consume the hyenas, suggesting that the kills serve to reduce competition rather than to provide nutrition. Spotted hyenas can drive lionesses from their kills, however, if they outnumber them by at least four to one. Lions also regularly kill wild dogs, accounting for $30-50 \%$ of all deaths of pups and adults. Hyenas, in contrast, successfully steal food from wild dogs $50-60 \%$ of the time. Cheetahs also lose $12-14 \%$ of their kills to hyenas, and lose many of their pups to predation by lions, who do not eat the pups (Van Valkenburgh, 2001).

Studies of the north American carnivore guild paint a similar picture (Van Valkenburgh, 2001). Three years after 35 gray wolves were reintroduced to Yellowstone National Park, for example, they tripled their numbers while coyote numbers plummeted. Numerous wolfkilled coyotes were discovered, often uneaten, and coyote pups were also killed. Wolves 
and Grizzly bears were present at $27 \%$ and $36 \%$ of puma kills, respectively, and were responsible for three and one puma deaths. Grizzly bears and wolves will also both occasionally prey on hibernating adult black bears, digging them out of their dens and feeding on them. Pumas kill coyotes and bobcats, who often scavenge puma kills, whereas coyotes are responsible for 50-87\% of kit and swift fox deaths. Van Valkenburgh (2001) summarizes interspecific interactions among sympatric predators in modern environments (p. 109):

First, most interspecific interactions between predators occur as contests for the possession of a kill. The motivation for intraguild predation appears to be hunger in many instances, particularly when the body size difference between the two species is fairly large (e.g., coyote-kit fox, brown bear-black bear, lynx-red fox). However, equally or more often, the victim is not eaten, and the likely motivation is to remove a competitor who might also prey on the agressor's young. Second, body size is the usual determinant of rank within the guild; larger species tend to dominate smaller ones (e.g., lion-hyena, hyena-wild dog, brown bear-wolf, wolfcoyote, tiger-leopard, jaguar-puma). Third, the body size rule can be overturned by the smaller species acting as a group (e.g., hyenas versus lions, wolves versus bears, wild dogs versus hyenas). Fourth, intraguild predation and kleptoparasitism occur in both forested and open environments.

Interspecific conflicts include humans - many people are killed by large carnivores, and humans decimate carnivore populations. A study of Ugandan Game Department archives from 1923-1994 found records of 393 attacks on humans by large carnivores, 247 (63\%) of which were fatal. Most fatalities were from lions, followed by leopards (Treves \& Naughton-Treves, 1999). A review of the literature on human-felid conflicts found that felid body masses roughly equal to or greater than humans were strongly positively associated with the degree of conflict (Inskip \& Zimmermann, 2009).

Turning to the Plio-Pleistocene environment, early Homo would have been potential prey for numerous carnivores, many two to four times as large as Homo, and several that would have hunted in packs. Compared to today, Plio-Pleistocene East African herbivore communities included many more megaherbivores (>1000kg), browsing on C3 vegetation, and the large $(>100 \mathrm{~kg})$ carnivores that preyed on them, such as giant hyenas, sabertooth cats, lions, and highly carnivorous bears (Faith, Rowan, \& Du, 2019; Treves \& Palmqvist, 2007). Early Homo would have fought with these carnivores for carcasses and plausibly been targeted by them as competitors. See Figure 1. Many authors have therefore concluded that greater carnivory by early Homo would only have been possible with joint predator defense provided by a group of highly cooperative males that probably used weapons of some sort (e.g., Bickerton \& Szathmáry, 2011; DeVore \& Washburn, 1963; Treves \& Palmqvist, 2007; Van Valkenburgh, 2001; Willems \& van Schaik, 2017). 


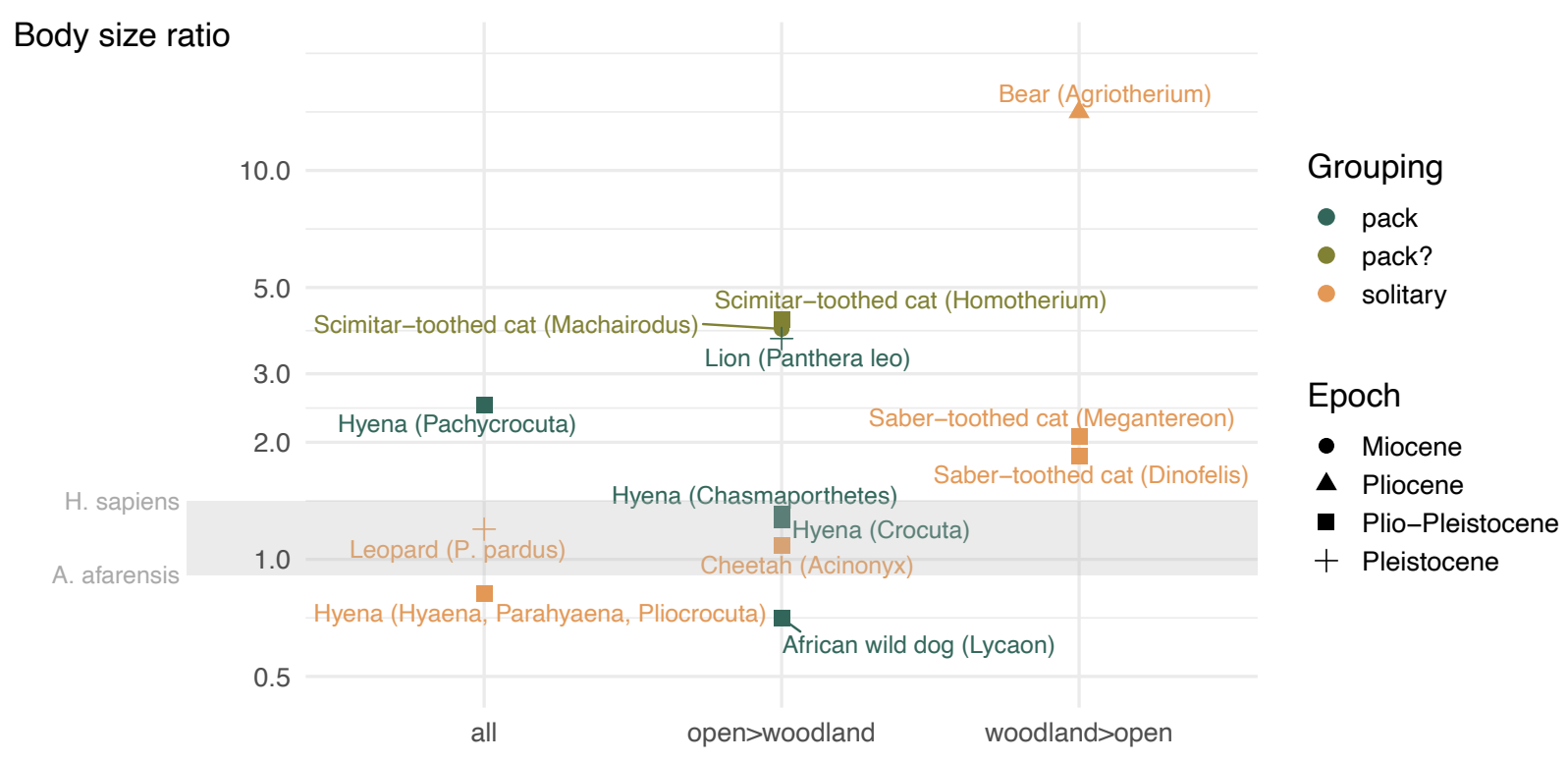

Figure 1: African carnivore species (extinct and extant) that overlapped with Homo and other hominins, by body size, habitat, pack hunting, and epoch. Body size ratio is the ratio of the mass of the carnivore relative to that of Homo erectus ( $46 \mathrm{~kg}$ ), displayed log-scaled. The gray rectangle indicates the range of body sizes of hominins relative to H. erectus. Data from Treves \& Palmqvist (2007).

\section{Aposematic and other predator-prey signals}

Counterintuitively, many prey species have evolved conspicuous coloration, and when predators approach exhibit striking visual and auditory behaviors. The more common response to predation is to evolve camouflage and other forms of crypsis, of course (Ruxton, Allen, Sherratt, \& Speed, 2018), and to mitigate the cost of predators eavesdropping on mating and other signals (Goodale, Ruxton, \& Beauchamp, 2019; Magnhagen, 1991). Singing bird and primate species occupy safer arboreal habitats rather than terrestrial habitats, for instance (Schruth \& Jordania, 2020).

Nevertheless, prey can benefit by signaling predators because they have a shared interest: predators in not chasing prey they can't catch, kill, or safely eat, and prey in not being chased or eaten. This has resulted in the evolution of numerous predator-prey signaling systems, such as the distinctive coloration in monarch butterflies that indicates their unpalatability, a phenomenon termed aposematism. Warning signals also include smells, sounds, and various behaviors that convey a broad array of defenses beyond unpalatability, such as stings, venomous fangs, formidability, detection of the predator, and ability to escape. Such signals, like the black and yellow bands of stinging bees and hornets, and auditory signals like the rattlesnake's rattle, have evolved in a wide range of taxa (Caro, 2005; Caro \& Allen, 2017; Caro \& Ruxton, 2019). Despite the frequent evolution of mimics, aposematic signals generally appear to be honest (Summers, Speed, Blount, \& Stuckert, 2015). 


\section{Pursuit deterrence}

The vast majority of carnivores - $80 \%$ to $95 \%$ - are solitary hunters, either pursuing prey (e.g., cheetahs, commonly foxes or lynx) or ambushing them (e.g., tigers, jaguars, cougars, and leopards). Only a few terrestrial mammalian predators, such as lions and wolves, consistently hunt in groups, though some, such as hyenas, occasionally do (Hirt, Tucker, Müller, Rosenbaum, \& Brose, 2020). Pursuit hunters target smaller, slower prey that they can catch and subdue; group hunters are able to subdue larger slow prey; and ambush hunters can catch a wider range of prey, including faster and larger prey (Hirt et al., 2020).

Upon detecting a predator, a number of prey species signal with white rumps, tail positions, distinctive gaits, or auditory calls. Whether these signals are directed to the predator, conspecifics, or both is still debated for many species. Signals directed at conspecifics function as alarm calls. Signals directed at the predator could inform it that it has been detected (perception advertisement), which reduces its odds of success, especially for ambush predators, or that the prey has a superior ability to escape (quality advertisement), which reduces its odds of being caught. Perception and quality signals would deter the predator from pursuing the prey (Bergstrom \& Lachmann, 2001; Caro \& Allen, 2017; Ramesh \& Mitchell, 2018; Summers et al., 2015). Examples of pursuit deterrence signals include birds and lagomorphs living in open habitats that display white tails; examples of quality advertisements include artiodactyls with thick dark stripes that amplify stotting or leaping behaviors, and bird songs that deter pursuing raptors (Caro \& Allen, 2017; Cresswell, 1994; Hedley \& Caro, 2021).

There is increasing evidence that alarm calls, common in many species, also function as pursuit deterrence signals. The evolution of alarm calls is puzzling because they are a public good: they reduce listeners' risk of predation but are thought to increase the signaler's risk, therefore requiring, e.g., kin selection or reciprocal altruism to evolve. But alarm calls also signal to the predator that it has been detected, which might deter its pursuit, directly benefiting the signaler (Bergstrom \& Lachmann, 2001; Ramesh \& Mitchell, 2018; Woodland, Jaafar, \& Knight, 1980). Many studies now show that alarm calls do deter predators (Hedley \& Caro, 2021; Ostreiher \& Heifetz, 2020; Woodland et al., 1980). When many monkey species detect leopards, for example (an ambush predator), they give alarm calls. A study that tracked a radio-collared leopard found that shortly after monkey alarm calls, the leopard left (Zuberbühler, Jenny, \& Bshary, 1999). A study of vervet monkeys using camera traps and GPS collars similarly found that when monkeys called, leopards that had been moving toward them moved away (Isbell \& Bidner, 2016). An experimental study that tracked ocelots before and after playback of the alarm calls of titi and saki monkeys vs. their non-alarm calls, found that ocelots stayed hidden in response to nonalarm calls but after alarm calls they moved away (Adams \& Kitchen, 2018). Similar patterns are seen in non-primates. A two-year observational study of squirrels, for instance, found that vocalizations served a dual function of deterring predators and warning offspring (Burnett \& Koprowski, 2020). Predator harassment, termed mobbing, is also widespread across species and typically involves vocalizations (Carlson \& Griesser, 2021). 


\section{Pelage coloration and defensive capabilities in carnivores and other mammals}

Many mammals have aposematic coloring - black and white skunks and their noxious anal secretions are a well-known example. Studies of pelage coloration in terrestrial carnivores and other mammals, along with their use of defensive anal glands and spines, lifestyle, body size, and habitat, found that bold contrasting coloration is generally a clear and honest signal of defensive capabilities and behavioral pugnacity (Howell et al., 2021; Stankowich, Caro, \& Cox, 2011). One study of terrestrial carnivores found five antipredator strategies, four of which relied on various forms of crypsis. The fifth strategy, which evolved independently several times, comprised smaller or mid-sized carnivores living in open habitats who are slow and stocky (e.g., striped skunks, polecats, badgers), and so must defend themselves and signal this ability from long distances (Stankowich et al., 2011).

Hominins, like the mid-sized carnivores exhibiting the fifth strategy, evolved in an open habitat and were smaller and slower than many predators (see Figures 1 and 2). Hence, there might have been a selection pressure on hominins, especially the more carnivorous Homo, to evolve a long-distance aposematic signal. Although body paint and other decorations, which are common across cultures (Schildkrout, 2004), might have served this function, here the focus will be on the possibility that synchronized auditory and visual signals provided hominins with a long-distance signal of pugnacious formidability.

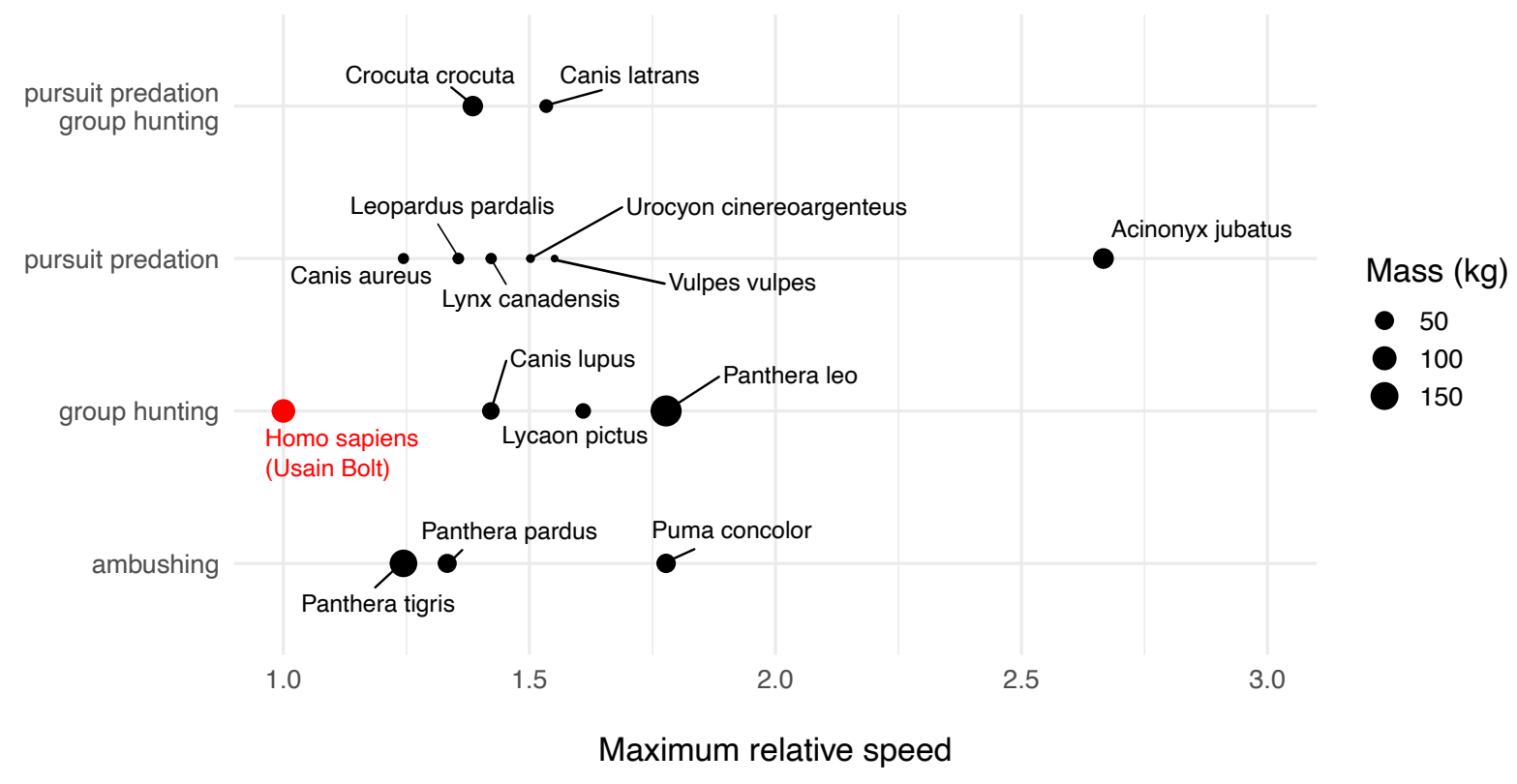

Figure 2: Extant carnivore maximum sprint speeds, relative to the maximun modern human sprint speed (45 km/hr), by hunting strategy. Data from Hirt et al. (2020).

\section{Synchronized signaling by prey to predators}

A few social species exhibit highly synchronized signaling to predators. Giant honeybees, for example, nest in the open and are preyed on by birds, hornets, and other animals. When hornets approach the nest, bees on the surface initiate a highly coordinated "shimmering" or wave by raising their abdomens, which deters hornet attacks (Kastberger, Schmelzer, \& 
Kranner, 2008). See Figure 3. When hornets persist in attack, numerous bees can engulf it in a ball and kill it by activating their flight muscles to increase core temperature to 45C, which is lethal to hornets (a strategy known as heat balling). Shimmering is therefore probably an aposematic signal that the colony has detected the hornet and will counterattack with a heat ball if it does not withdraw (Kastberger et al., 2008).
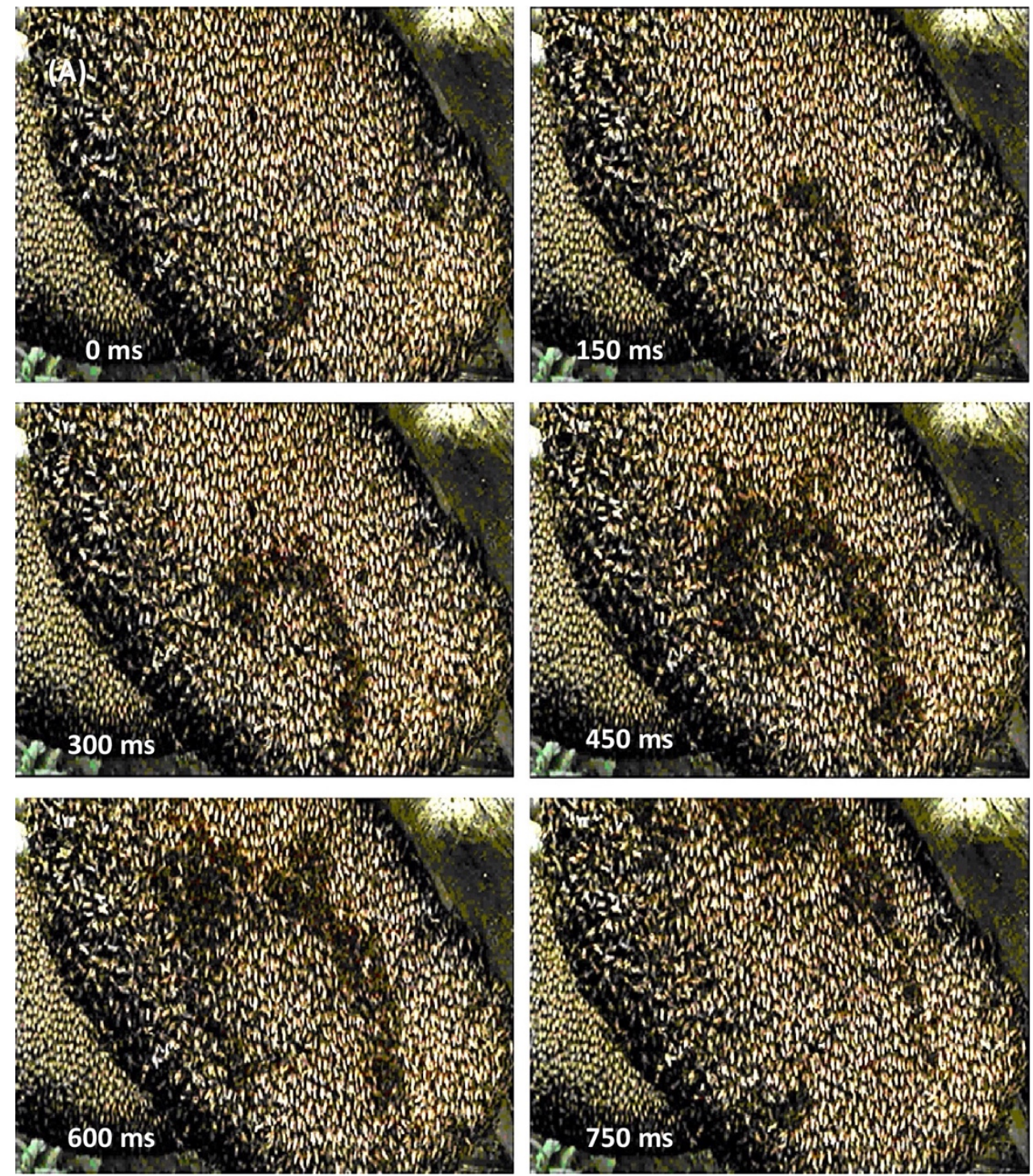

Figure 3: An image sequence, with intervals of $150 \mathrm{~ms}$, showing the propagation of a spiral wave in a colony of giant honeybees (Apis dorsata) (Figure from by Kastberger et al. 2008).

Asian honeybees exhibit synchronized aposematic signals too, such as hisses, which "are produced when many workers move their bodies and vibrate their wings synchronously in response to mechanical disturbance or predator attack, including harassment by hornets" (Mattila et al., 2021, p. 3). When predatory hornets approach the nest, guard bees at the entrance also simultaneously vibrate their abdomens from side to side for a few seconds, 
which is visually striking and is accompanied by a loud buzzing noise. The display honestly signals the number of nest defenders, reduces hornet approach to the nest, and reduces bee predation (Dong, Tan, \& Nieh, 2021; Tan et al., 2012).

In response to avian attacks, schools of sulphur molly fish engage in surface waves lasting for up to two minutes that are highly conspicuous, repetitive, and rhythmic, involving many thousands of individuals. Waves double the time birds wait until their next attack thereby substantially reducing attack frequency, and, in one avian predator, capture probability. The attack delay could be a result of a confusion effect or a consequence of waves acting as a perception advertisement (Doran et al., 2021).

Gibbons, monogamous apes that are relatively close human relatives, sing highly synchronized male-female duets that have territorial and other functions. White-handed gibbons also sing predator-specific songs in response to tigers and leopards, ambush predators, but not other predators (Clarke, Reichard, \& Zuberbühler, 2006, 2012). Although these songs clearly serve as alarm calls to other gibbons, they also function to deter predators, perhaps by advertising to ambush predators that they have been detected (Andrieu et al., 2020).

\section{Coalition quality signals and predator deterrence}

One evolutionary hypothesis for the biological origins of highly synchronized group performances of music and dance is that they evolved from territorial advertisements (Hagen \& Bryant, 2003; Hagen \& Hammerstein, 2009; Mehr et al., 2021). In brief, in many species, including apes and other primates, territory owners advertise ownership with prominent auditory and/or visual displays that serve to warn off intruders. In social species that defend territories in groups, such as chimpanzees and group-hunting wolves, lions, and other carnivores, these displays involve coordinated vocal signals such as group howling and roaring. In mated pairs of birds and primates, territorial advertisements often involve highly synchronized duets by the mated pair. Coordinated calls are credible signals that the territory is defended by multiple individuals. In highly synchronized calls, the degree of synchronization is a credible signal of coalition quality: synchronization requires time-consuming practice among cooperating individuals. Greater degrees of synchronization therefore credibly indicate more extensive periods of cooperation, and thus more formidable group defense against threats. For detailed expositions of the credible coalition quality signaling hypothesis and supporting evidence, see Hagen \& Bryant (2003), Hagen \& Hammerstein (2009), and Mehr et al. (2021).

The credible coalition quality signaling hypothesis extends naturally to predator deterrence signaling. Highly synchronized visual and auditory displays by hominins, similar to those in honeybees and gibbons, would have credibly signaled to the predator that it had been detected and, as in the conspicuous markings of many pugnacious carnivores, that any attack would be met with a fierce and effective response by a high quality coalition. Jordania (2008), who argued for the role of increased predation pressure on hominins and predator signaling in the evolution of rhythm, put it nicely (p. 61): 
syncronised group shouting gives a strong message to the predator that it has to deal not with the group of scared individs, but with a well-organised and determined unity. [typos in the original]

There is indeed evidence that hunter-gatherers and forest peoples use trance dancing, rhythmic clapping, drumming, chanting, and singing to ward off wild animals (reviewed in Knight \& Lewis, 2017).

\section{Hominins as a lethal threat to other hominins}

As Homo evolved into a hunting species, the distinction between territorial and predator signaling might have blurred. Homo could have become Homo's major predator. Much wolf mortality, for example, is from other wolves (Barber-Meyer, Wheeldon, \& Mech, 2021; Mech \& Barber-Meyer, 2017). Significant mortality from conspecifics might have occurred even earlier in human evolution: coalitions of chimpanzees kill other chimpanzees, raising the possibility that the last common ancestor of humans and chimpanzees engaged in coalitional killing of conspecifics (for a review of the contentious issue of warfare in the human lineage, see Kissel \& Kim, 2019). More generally, territorial conflicts and territorial signaling are common between species (Drury, Cowen, \& Grether, 2020; Grether, Peiman, Tobias, \& Robinson, 2017). Hence, territorial and predator signaling in hominin lineages could have deterred intruders and predators from the same species or competing species.

\section{Within-group signaling functions}

In primates and other animals, there appears to be substantial overlap between predator signals and alarm calls (reviewed earlier). Alarm calls, which function to warn fellow group members of a predator threat and inform the predator that it has been detected, can also serve to recruit defenders. In Asian honeybees, for example, hornet predators, especially group-hunting hornet species, trigger frenetic within-colony antipredator signaling that recruits more defenders (Mattila et al., 2021). Recruitment calls are also seen in birds and mammals (Furrer \& Manser, 2009; Woods, Kings, McIvor, \& Thornton, 2018). Hagen \& Bryant (2003) proposed that in humans, work songs, which are common across cultures, function in part to recruit more workers. Hominin defense signals might similarly have functioned to recruit more defenders.

Predator signaling could also have selected for signal variation. Different predators, such as eagles, leopards, lions, and hyenas, had different body masses and hunting strategies, such as ambush, pack, and pursuit, that would have required different defensive responses. Some primate species have therefore evolved predator-specific alarm calls (Boë et al., 2019; Fitch, 2005; Schlenker, Chemla, \& Zuberbühler, 2016). It is possible that if hominins evolved antipredator signals that they were specific to different predators. Predatorspecific calls in primates, which many have argued were precursors for the evolution of human language (for review, see Fischer \& Price, 2017), could have also have selected for, e.g., a combinatorial system of signal generation that would be one of the biological roots of music variation in modern humans. In white-handed gibbons, for instance, duet songs, which serve territorial defense and other functions, are similar to predator songs, which are sung when a predator is detected. Nevertheless, gibbons readily discriminate between 
the two songs, engaging in vigilance behaviors following predator songs but never following duet songs (Andrieu et al., 2020). Diverse predator threats might have been one selection pressure for signal variation in Homo.

\section{Predator deception and confusion}

Many flocks of birds and schools of fish exhibit striking collective responses to predators. One popular interpretation of such collective behavior is that it overwhelms predator visual systems, which cannot accurately track specific prey in a swarm, increasing prey survival, a phenomenon termed predator confusion. Several theoretical models investigate how different constraints on predator visual processing and different prey response rules might generate the observed collective patterns of behavior (Krakauer, 1995; Olson, Hintze, Dyer, Knoester, \& Adami, 2013; Olson, Hintze, Dyer, Moore, \& Adami, 2016). One model that reproduced several common prey aggregation patterns found that swarming did not protect prey from predators, however, implying that some other benefit was required to explain it (Chen \& Kolokolnikov, 2014). Empirically, studies generally support the existence of predator confusion, albeit with several negative results. In a meta-analysis of 25 predator-prey systems, for example, there was evidence of predator confusion in 16 (64\%, Jeschke \& Tollrian, 2007).

Some authors have linked the evolution of human music and dance to predator confusion and deception. Ehrenreich (2007) (p. 28), for example, writes:

Like primates in the wild today, early humans probably faced off predatory animals collectively-banding together in a tight group, stamping their feet, shouting, and waving sticks or branches....At some point, early humans or hominids may have learned to synchronize their stampings and stick-wavings in the face of a predator, and the core of my speculation is that the predator might be tricked by this synchronous behavior into thinking that it faced-not a group of individually weak and defenseless humans-but a single, very large animal.

Knight \& Lewis (2017) (p. 437) similarly write:

Lions prowling in the dark may have been more wary of approaching a noisy bunch of females and infants if unexpected pitch variations made it difficult to estimate group size and risk.

Schruth \& Jordania (2020) (p. 11) likewise propose that group auditory performances involve deceptive rather than credible signals of group size:

On the one hand, singing was likely a safety hazard-particularly in terrestrial environments, rich in predators. On the other hand, singing-particularly group singing and loud choruses-could have also benefited practitioners as a type of weaponized acoustic instrument for protection from predators, particularly upon terrestrial visitations, chiefly via an elevation-descent form of the aforementioned Beau Geste effect. [emphasis added] 
The Beau Geste effect refers to a single territorial individual using a large song repertoire to deceive listeners into believing that multiple individuals are present (Krebs, 1977).

Deception is rife in nature, including during encounters between predators and prey (Caro \& Ruxton, 2019), so these proposals are worth considering. In hunter-gatherers, for example, disguises are often used for hunting and warfare (Buckner, 2021). Unlike the shared interest among predators and prey in the credible signaling hypothesis, however, deceptive strategies involve conflicts between predators and prey, and predators would be under selection to evolve resistance to deception. Nevertheless, both honest and deceptive predator signaling might help explain the evolution of coordinated vocalizations in Homo.

\section{Future research}

Much remains to be done to test the coalition quality hypothesis. One priority is to evaluate the cross-cultural evidence for the role of music in territorial defense, alliance formation, and predator defense, especially in hunter-gatherer societies. The Human Area Relations Files (HRAF), a database of ethnographic records on 360 cultures, including 88 huntergatherer cultures, currently has documents that discuss music in the context of warfare from 122 cultures, including 37 hunter-gatherer cultures, and documents that discuss music in the situations in which alliances are formed, such as visiting, hospitality, and other forms of intergroup relations, from 237 cultures, including 54 hunter-gatherer cultures. It also has documents that discuss music and marriages, which are relevant to the proposal that alliance-by-marriage unifies the coalition quality and sexual selection hypotheses (Hagen and Bryant, 2003), from 189 cultures, including 35 hunter-gatherer cultures. The extent to which these documents support or refute the coalition quality hypothesis must be determined by future research.

Another priority is to determine if there are universal psychological mechanisms to, for example, motivate members of groups to perform highly synchronized songs and dances for visiting groups, and for visitors to evaluate the quality of these performances when deciding whether to form cooperative intergroup relationships, including marriage alliances. Do similar universal psychological mechanisms exist to motivate energetic performances of noisy synchronized audiomotor displays and perhaps aggressive songs and dances when groups encounter predators or enemies?

\section{Concluding remarks}

In the Pliocene, hominins evolved an increasingly committed terrestrial lifestyle in open habitats that probably exposed them to increased predation pressure from Africa's formidable predator guild. In the Pleistocene, Homo transitioned to a more carnivorous lifestyle that would have further increased predation pressure. Many authors have therefore proposed that an effective defense against predators would have required a high degree of cooperation by the smaller and slower hominins. Because it is in the interest of predators and potential prey to avoid encounters that will be costly for both, a wide variety of species, including carnivores and apes and other primates, have evolved visual and 
auditory signals that deter predators by credibly signaling detection and/or the ability to effectively defend themselves. The credible coalition quality signaling hypothesis (Hagen \& Bryant, 2003) extends naturally to signaling detection and defensive capabilities to external threats, be they predators (Jordania, 2008) or conspecifics, so as to deter their attacks. In some cooperative species, such as honeybees and gibbons, territorial and predator deterrent signals indeed involve highly synchronized visual and auditory displays among group members. It is plausible that, like their fellow apes, increasingly cooperative hominins evolved increasingly synchronized visual and auditory displays to warn fellow group members of specific deadly threats and recruit defenders, to credibly signal predators or hominin attackers that they had been detected and would be met with a highly coordinated defensive response, and to signal competing intra- or interspecific groups that the territory was occupied and well-defended by a highly cooperative group. Like gibbons, hominins might have evolved to produce distinct signals for territorial advertisements vs. predator detection. The evolved cognitive abilities underlying these behaviors were foundations for the later evolution of fully human music and dance, which involved coalitions displaying their quality to their allies as much as to their enemies (Hagen \& Bryant, 2003; Hagen \& Hammerstein, 2009; Mehr et al., 2021).

\section{Conflicts of interest}

The author has no relevant financial or non-financial interests to disclose. 


\section{References}

Adams, D. B., \& Kitchen, D. M. (2018). Experimental evidence that titi and saki monkey alarm calls deter an ambush predator. Animal Behaviour, 145, 141-147.

https://doi.org/10.1016/j.anbehav.2018.09.010

Almécija, S., Hammond, A. S., Thompson, N. E., Pugh, K. D., Moyà-Solà, S., \& Alba, D. M. (2021). Fossil apes and human evolution. Science, 372(6542), eabb4363.

https://doi.org/10.1126/science.abb4363

Andrieu, J., Penny, S. G., Bouchet, H., Malaivijitnond, S., Reichard, U. H., \& Zuberbühler, K. (2020). White-handed gibbons discriminate context-specific song compositions. PeerJ, 8 , e9477. https://doi.org/10.7717/peerj. 9477

Antón, S. C., Potts, R., \& Aiello, L. C. (2014). Evolution of early Homo : An integrated biological perspective. Science, 345(6192), 1236828.

https://doi.org/10.1126/science.1236828

Barber-Meyer, S. M., Wheeldon, T. J., \& Mech, L. D. (2021). The importance of wilderness to wolf (canis lupus) survival and cause-specific mortality over 50 years. Biological Conservation, 258, 109145. https://doi.org/10.1016/j.biocon.2021.109145

Ben-Dor, M., Sirtoli, R., \& Barkai, R. (2021). The evolution of the human trophic level during the Pleistocene. American Journal of Physical Anthropology.

https://doi.org/10.1002/ajpa.24247

Bergstrom, C. T., \& Lachmann, M. (2001). Alarm calls as costly signals of antipredator vigilance: The watchful babbler game. Animal Behaviour, 61(3), 535-543.

https://doi.org/10.1006/anbe.2000.1636

Bickerton, D., \& Szathmáry, E. (2011). Confrontational scavenging as a possible source for language and cooperation. BMC Evolutionary Biology, 11(1), 261.

https://doi.org/10.1186/1471-2148-11-261

Boë, L.-J., Sawallis, T. R., Fagot, J., Badin, P., Barbier, G., Captier, G., ... Schwartz, J.-L. (2019). Which way to the dawn of speech?: Reanalyzing half a century of debates and data in light of speech science. Science Advances. https://doi.org/10.1126/sciadv.aaw3916

Buckner, W. (2021). Disguises and the origins of clothing. Human Nature, 32(4), 706-728. https://doi.org/10.1007/s12110-021-09415-7

Burnett, A. D., \& Koprowski, J. L. (2020). Ultimate causes of antipredator vocalizations in a nonhibernating squirrel. Animal Behaviour, 168, 225-230.

https://doi.org/10.1016/j.anbehav.2020.08.016

Carlson, N. V., \& Griesser, M. (2021). Mobbing in animals: A thorough review and proposed future directions. https://doi.org/10.32942/osf.io/rty9q

Caro, T. (2005). Antipredator defenses in birds and mammals. University of Chicago Press. 
Caro, T., \& Allen, W. L. (2017). Interspecific visual signalling in animals and plants: A functional classification. Philosophical Transactions of the Royal Society B: Biological Sciences, 372(1724), 20160344. https://doi.org/10.1098/rstb.2016.0344

Caro, T., \& Ruxton, G. (2019). Aposematism: Unpacking the defences. Trends in Ecology \& Evolution, 34(7), 595-604. https://doi.org/10.1016/j.tree.2019.02.015

Chen, Y., \& Kolokolnikov, T. (2014). A minimal model of predatorswarm interactions. Journal of The Royal Society Interface, 11(94), 20131208.

https://doi.org/10.1098/rsif.2013.1208

Clarke, E., Reichard, U. H., \& Zuberbühler, K. (2006). The syntax and meaning of wild gibbon songs. PLOS ONE, 1(1), e73. https://doi.org/10.1371/journal.pone.0000073

Clarke, E., Reichard, U. H., \& Zuberbühler, K. (2012). The anti-predator behaviour of wild white-handed gibbons (Hylobates lar). Behavioral Ecology and Sociobiology, 66(1), 85-96. https://doi.org/10.1007/s00265-011-1256-5

Coleman, B. T., \& Hill, R. A. (2014). Living in a landscape of fear: The impact of predation, resource availability and habitat structure on primate range use. Animal Behaviour, 88, 165-173. https://doi.org/10.1016/j.anbehav.2013.11.027

Couvreur, T. L. P., Dauby, G., Blach-Overgaard, A., Deblauwe, V., Dessein, S., Droissart, V., ... Sepulchre, P. (2021). Tectonics, climate and the diversification of the tropical african terrestrial flora and fauna. Biological Reviews, 96(1), 16-51.

https://doi.org/10.1111/brv.12644

Couzin, I. D. (2018). Synchronization: The key to effective communication in animal collectives. Trends in Cognitive Sciences, 22(10), 844-846.

https://doi.org/10.1016/j.tics.2018.08.001

Creel, S., \& Christianson, D. (2008). Relationships between direct predation and risk effects. Trends in Ecology \& Evolution, 23(4), 194-201. https://doi.org/10.1016/j.tree.2007.12.004

Cresswell, W. (1994). Song as a pursuit-deterrent signal, and its occurrence relative to other anti-predation behaviours of skylark (Alauda arvensis) on attack by merlins (Falco columbarius). Behavioral Ecology and Sociobiology, 34(3), 217-223.

DeVore, I., \& Washburn, S. L. (1963). Baboon ecology and human evolution. In African ecology and human evolution. Routledge.

Domínguez-Rodrigo, M., Baquedano, E., Organista, E., Cobo-Sánchez, L., Mabulla, A., Maskara, V., ... González-Aguilera, D. (2021). Early Pleistocene faunivorous hominins were not kleptoparasitic, and this impacted the evolution of human anatomy and socio-ecology. Scientific Reports, 11(1), 16135. https://doi.org/10.1038/s41598-021-94783-4

Dong, S., Tan, K., \& Nieh, J. C. (2021). Visual contagion in prey defence signals can enhance honest defence. Journal of Animal Ecology, 90(3), 594-601. https://doi.org/10.1111/13652656.13390 
Doran, C., Bierbach, D., Lukas, J., Klamser, P., Landgraf, T., Klenz, H., ... Krause, J. (2021). Fish waves as emergent collective antipredator behavior. Current Biology, S0960982221016547. https://doi.org/10.1016/j.cub.2021.11.068

Drury, J. P., Cowen, M. C., \& Grether, G. F. (2020). Competition and hybridization drive interspecific territoriality in birds. Proceedings of the National Academy of Sciences, 117(23), 12923-12930. https://doi.org/10.1073/pnas.1921380117

Ehrenreich, B. (2007). Dancing in the streets: A history of collective joy. Macmillan.

Eriksson, O. (2016). Evolution of angiosperm seed disperser mutualisms: The timing of origins and their consequences for coevolutionary interactions between angiosperms and frugivores. Biological Reviews, 91(1), 168-186. https://doi.org/10.1111/brv.12164

Faith, J. T., Rowan, J., \& Du, A. (2019). Early hominins evolved within non-analog ecosystems. Proceedings of the National Academy of Sciences, 116(43), 21478-21483.

https://doi.org/10.1073/pnas.1909284116

Finch, C. E., \& Stanford, C. B. (2004). Meat-adaptive genes and the evolution of slower aging in humans. The Quarterly Review of Biology, 79(1), 3-50. https://doi.org/10.1086/381662

Fischer, J., \& Price, T. (2017). Meaning, intention, and inference in primate vocal communication. Neuroscience \& Biobehavioral Reviews, 82, 22-31.

https://doi.org/10.1016/j.neubiorev.2016.10.014

Fitch, W. T. (2005). The evolution of language: A comparative review. Biology \& Philosophy, 20(2-3), 193-203. https://doi.org/10.1007/s10539-005-5597-1

Foley, R. A. (2016). Mosaic evolution and the pattern of transitions in the hominin lineage. Philosophical Transactions of the Royal Society B: Biological Sciences, 371(1698), 20150244. https://doi.org/10.1098/rstb.2015.0244

Furrer, R. D., \& Manser, M. B. (2009). Banded mongoose recruitment calls convey information about risk and not stimulus type. Animal Behaviour, 78(1), 195-201. https://doi.org/10.1016/j.anbehav.2009.05.002

Gaynor, K. M., Brown, J. S., Middleton, A. D., Power, M. E., \& Brashares, J. S. (2019). Landscapes of fear: Spatial patterns of risk perception and response. Trends in Ecology \& Evolution, 34(4), 355-368. https://doi.org/10.1016/j.tree.2019.01.004

Goodale, E., Ruxton, G. D., \& Beauchamp, G. (2019). Predator eavesdropping in a mixedspecies environment: How prey species may use grouping, confusion, and the cocktail party effect to reduce predator detection. Frontiers in Ecology and Evolution, 7.

Grether, G. F., Peiman, K. S., Tobias, J. A., \& Robinson, B. W. (2017). Causes and consequences of behavioral interference between species. Trends in Ecology \& Evolution, 32(10), 760-772. https://doi.org/10.1016/j.tree.2017.07.004 
Hagen, E. H., \& Bryant, G. A. (2003). Music and dance as a coalition signaling system. Human Nature, 14(1), 21-51. https://doi.org/10.1007/s12110-003-1015-Z

Hagen, E. H., \& Hammerstein, P. (2009). Did neanderthals and other early humans sing? Seeking the biological roots of music in the territorial advertisements of primates, lions, hyenas, and wolves. Musicae Scientiae, 13(2_suppl), 291-320.

https://doi.org/10.1177/1029864909013002131

Hall, M. L. (2009). A review of vocal duetting in birds. In M. Naguib, V. M. Janik, K.

Zuberbuhler, \& N. S. Clayton (Eds.), Advances in the study of behavior (Vol. 40, pp. 67-121). https://doi.org/10.1016/S0065-3454(09)40003-2

Hart, D., \& Sussman, R. W. (2008). Man the hunted: Primates, predators, and human evolution. Routledge.

Hedley, E., \& Caro, T. (2021). Aposematism and mimicry in birds. Ibis. https://doi.org/10.1111/ibi.13025

Hirt, M. R., Tucker, M., Müller, T., Rosenbaum, B., \& Brose, U. (2020). Rethinking trophic niches: Speed and body mass colimit prey space of mammalian predators. Ecology and Evolution, 10(14), 7094-7105. https://doi.org/10.1002/ece3.6411

Howell, N., Sheard, C., Koneru, M., Brockelsby, K., Ono, K., \& Caro, T. (2021). Aposematism in mammals. Evolution, 75(10), 2480-2493. https://doi.org/10.1111/evo.14320

Hunt, K. D. (2016). Why are there apes? Evidence for the co-evolution of ape and monkey ecomorphology. Journal of Anatomy, 228(4), 630-685. https://doi.org/10.1111/joa.12454

Inskip, C., \& Zimmermann, A. (2009). Human-felid conflict: A review of patterns and priorities worldwide. Oryx, 43(01), 18. https://doi.org/10.1017/S003060530899030X

Isbell, L. A., \& Bidner, L. R. (2016). Vervet monkey (chlorocebus pygerythrus) alarm calls to leopards (panthera pardus) function as a predator deterrent. Behaviour, 153(5), 591-606. https://doi.org/10.1163/1568539X-00003365

Isbell, L. A., Bidner, L. R., Van Cleave, E. K., Matsumoto-Oda, A., \& Crofoot, M. C. (2018). GPSidentified vulnerabilities of savannah-woodland primates to leopard predation and their implications for early hominins. Journal of Human Evolution, 118, 1-13. https://doi.org/10.1016/j.jhevol.2018.02.003

Jeschke, J. M., \& Tollrian, R. (2007). Prey swarming: Which predators become confused and why? Animal Behaviour, 74(3), 387-393. https://doi.org/10.1016/j.anbehav.2006.08.020

Jordania, J. (2008). Origins of rhythm and the defence strategy of human ancestors. In Problems of traditional polyphony. Materials of the third international symposium on traditional polyphony. Third international research centre of traditional polyphony, tbilisi state conservatory (pp. 55-66). 
Kastberger, G., Schmelzer, E., \& Kranner, I. (2008). Social waves in giant honeybees repel hornets. PLoS ONE, 3(9), e3141. https://doi.org/10.1371/journal.pone.0003141

Kaya, F., Bibi, F., Žliobaite, I., Eronen, J. T., Hui, T., \& Fortelius, M. (2018). The rise and fall of the old world savannah fauna and the origins of the african savannah biome. Nature Ecology \& Evolution, 2(2), 241-246. https://doi.org/10.1038/s41559-017-0414-1

Kissel, M., \& Kim, N. C. (2019). The emergence of human warfare: Current perspectives. American Journal of Physical Anthropology, 168(S67), 141-163. https://doi.org/10.1002/ajpa.23751

Knight, C., \& Lewis, J. (2017). Wild voices: Mimicry, reversal, metaphor, and the emergence of language. Current Anthropology, 58(4), 435-453. https://doi.org/10.1086/692905

Kotz, S. A., Ravignani, A., \& Fitch, W. T. (2018). The evolution of rhythm processing. Trends in Cognitive Sciences, 22(10), 896-910. https://doi.org/10.1016/j.tics.2018.08.002

Krakauer, D. C. (1995). Groups confuse predators by exploiting perceptual bottlenecks: A connectionist model of the confusion effect. Behavioral Ecology and Sociobiology, 36(6), 421-429.

Krebs, J. R. (1977). The significance of song repertoires: The beau geste hypothesis. Animal Behaviour, 25, 475-478. https://doi.org/10.1016/0003-3472(77)90022-7

Kristan, W. B. (2016). Early evolution of neurons. Current Biology, 26(20), R949-R954. https://doi.org/10.1016/j.cub.2016.05.030

Magnhagen, C. (1991). Predation risk as a cost of reproduction. Trends in Ecology \& Evolution, 6(6), 183-186. https://doi.org/10.1016/0169-5347(91)90210-0

Mattila, H. R., Kernen, H. G., Otis, G. W., Nguyen, L. T. P., Pham, H. D., Knight, O. M., \& Phan, N. T. (2021). Giant hornet ( Vespa soror ) attacks trigger frenetic antipredator signalling in honeybee (Apis cerana ) colonies. Royal Society Open Science, 8(11), 211215.

https://doi.org/10.1098/rsos.211215

Mech, L. D., \& Barber-Meyer, S. M. (2017). Seasonality of intraspecific mortality by gray wolves. Journal of Mammalogy, 98(6), 1538-1546.

https://doi.org/10.1093/jmammal/gyx113

Mehr, S. A., Krasnow, M. M., Bryant, G. A., \& Hagen, E. H. (2021). Origins of music in credible signaling. Behavioral and Brain Sciences, 44.

https://doi.org/10.1017/S0140525X20000345

Merker, B. H. (1999). Synchronous chorusing and the origins of music. Musicae Scientiae, 3, 59-73.

Merker, B. H., Madison, G. S., \& Eckerdal, P. (2009). On the role and origin of isochrony in human rhythmic entrainment. Cortex, 45(1), 4-17.

https://doi.org/10.1016/j.cortex.2008.06.011 
Monk, T., \& Paulin, M. G. (2014). Predation and the origin of neurones. Brain, Behavior and Evolution, 84(4), 246-261. https://doi.org/10.1159/000368177

Monteza-Moreno, C. M., Crofoot, M. C., Grote, M. N., \& Jansen, P. A. (2020). Increased terrestriality in a Neotropical primate living on islands with reduced predation risk. Journal of Human Evolution, 143, 102768. https://doi.org/10.1016/j.jhevol.2020.102768

Olson, R. S., Hintze, A., Dyer, F. C., Knoester, D. B., \& Adami, C. (2013). Predator confusion is sufficient to evolve swarming behaviour. Journal of The Royal Society Interface, 10(85), 20130305. https://doi.org/10.1098/rsif.2013.0305

Olson, R. S., Hintze, A., Dyer, F. C., Moore, J. H., \& Adami, C. (2016). Exploring the coevolution of predator and prey morphology and behavior. Proceedings of the Artificial Life Conference 2016, 250-257. https://doi.org/10.7551/978-0-262-33936-0-ch045

Ostreiher, R., \& Heifetz, A. (2020). The function of sentinel alarm calls in the arabian babbler. Journal of Avian Biology, 51(10), jav.02513. https://doi.org/10.1111/jav.02513

Paulin, M. G., \& Cahill-Lane, J. (2019). Events in early nervous system evolution. Topics in Cognitive Science, tops.12461. https://doi.org/10.1111/tops.12461

Pobiner, B. L. (2020). The zooarchaeology and paleoecology of early hominin scavenging. Evolutionary Anthropology, 29(2), 68-82. https://doi.org/10.1002/evan.21824

Pontzer, H., \& Wood, B. M. (2021). Effects of evolution, ecology, and economy on human diet: Insights from hunter-gatherers and other small-scale societies. Annual Review of Nutrition, 41(1), annurev-nutr-111120-105520. https://doi.org/10.1146/annurev-nutr111120-105520

Püschel, H. P., Bertrand, O. C., O’Reilly, J. E., Bobe, R., \& Püschel, T. A. (2021). Divergencetime estimates for hominins provide insight into encephalization and body mass trends in human evolution. Nature Ecology \& Evolution, 5(6), 808-819.

https://doi.org/10.1038/s41559-021-01431-1

Ramesh, D., \& Mitchell, W. A. (2018). Evolution of signalling through pursuit deterrence in a two-prey model using game theory. Animal Behaviour, 146, 155-163.

https://doi.org/10.1016/j.anbehav.2018.10.012

Ravignani, A., Bowling, D. L., \& Fitch, W. T. (2014). Chorusing, synchrony, and the evolutionary functions of rhythm. Frontiers in Psychology, 5, 1118.

https://doi.org/10.3389/fpsyg.2014.01118

Reis, M. D., Gunnell, G. F., Barba-Montoya, J., Wilkins, A., Yang, Z., \& Yoder, A. D. (2018). Using phylogenomic data to explore the effects of relaxed clocks and calibration strategies on divergence time estimation: Primates as a test case. Systematic Biology, 67(4), 594-615. https://doi.org/10.1093/sysbio/syy001

Ruxton, G. D., Allen, W. L., Sherratt, T. N., \& Speed, M. P. (2018). Avoiding attack: The evolutionary ecology of crypsis, aposematism, and mimicry. Oxford University Press. 
Savage, P. E., Loui, P., Tarr, B., Schachner, A., Glowacki, L., Mithen, S., \& Fitch, W. T. (2021). Music as a coevolved system for social bonding. Behavioral and Brain Sciences, 44, e59. https://doi.org/10.1017/S0140525X20000333

Schildkrout, E. (2004). Inscribing the body. Annual Review of Anthropology, 33(1), 319-344. https://doi.org/10.1146/annurev.anthro.33.070203.143947

Schlenker, P., Chemla, E., \& Zuberbühler, K. (2016). What do monkey calls mean? Trends in Cognitive Sciences, 20(12), 894-904. https://doi.org/10.1016/j.tics.2016.10.004

Schruth, D. M., \& Jordania, J. (2020). Singing behavior via reduced predation risk. https://doi.org/10.31234/osf.io/u9m8z

Shultz, S., Opie, C., \& Atkinson, Q. D. (2011). Stepwise evolution of stable sociality in primates. Nature, 479(7372), 219-222. https://doi.org/10.1038/nature10601

Stankowich, T., Caro, T., \& Cox, M. (2011). Bold coloration and the evolution of aposematism in terrestrial carnivores. Evolution, 65(11), 3090-3099.

https://doi.org/10.1111/j.1558-5646.2011.01334.x

Steinthorsdottir, M., Coxall, H. K., Boer, A. M. de, Huber, M., Barbolini, N., Bradshaw, C. D., ... Strömberg, C. a. E. (2021). The miocene: The future of the past. Paleoceanography and Paleoclimatology, 36(4), e2020PA004037. https://doi.org/10.1029/2020PA004037

Summers, K., Speed, M. P., Blount, J. D., \& Stuckert, A. M. M. (2015). Are aposematic signals honest? A review. Journal of Evolutionary Biology, 28(9), 1583-1599.

https://doi.org/10.1111/jeb.12676

Sussman, R. W., Tab Rasmussen, D., \& Raven, P. H. (2013). Rethinking primate origins again. American Journal of Primatology, 75(2), 95-106. https://doi.org/10.1002/ajp.22096

Tan, K., Wang, Z., Li, H., Yang, S., Hu, Z., Kastberger, G., \& Oldroyd, B. P. (2012). An “i see you” prey-predator signal between the asian honeybee, apis cerana, and the hornet, vespa velutina. Animal Behaviour, 83(4), 879-882.

https://doi.org/10.1016/j.anbehav.2011.12.031

Treves, A., \& Naughton-Treves, L. (1999). Risk and opportunity for humans coexisting with large carnivores. Journal of Human Evolution, 36(3), 275-282.

https://doi.org/10.1006/jhev.1998.0268

Treves, A., \& Palmqvist, P. (2007). Reconstructing hominin interactions with mammalian carnivores (6.0-1.8 ma). In S. L. Gursky \& K. A. I. Nekaris (Eds.), Primate anti-predator strategies (pp. 355-381). https://doi.org/10.1007/978-0-387-34810-0_17

Van Valkenburgh, B. (2001). The dog-eat-dog world of carnivores: A review of past and present carnivore community dynamics. In C. B. Stanford, H. T. Bunn, R. L. Ciochon, \& B. A. Wood (Eds.), Meat-eating and human evolution (p. 21). 
Willems, E. P., \& Hill, R. A. (2009). Predator-specific landscapes of fear and resource distribution: Effects on spatial range use. Ecology, 90(2), 546-555.

https://doi.org/10.1890/08-0765.1

Willems, E. P., \& van Schaik, C. P. (2017). The social organization of Homo ergaster: Inferences from anti-predator responses in extant primates. Journal of Human Evolution, 109, 11-21. https://doi.org/10.1016/j.jhevol.2017.05.003

Woodland, D. J., Jaafar, Z., \& Knight, M.-L. (1980). The "pursuit deterrent" function of alarm signals. The American Naturalist, 115(5), 748-753. https://doi.org/10.1086/283596

Woods, R. D., Kings, M., McIvor, G. E., \& Thornton, A. (2018). Caller characteristics influence recruitment to collective anti-predator events in jackdaws. Scientific Reports, 8(1), 7343. https://doi.org/10.1038/s41598-018-25793-y

Zuberbühler, K., Jenny, D., \& Bshary, R. (1999). The predator deterrence function of primate alarm calls. Ethology, 105(6), 477-490. https://doi.org/10.1046/j.1439-

0310.1999.00396.x 